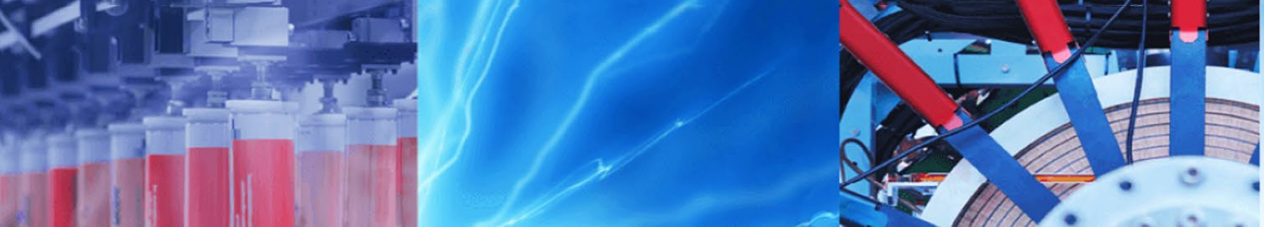

Research Article

\title{
Cross-layer unequal error protection using 64-HQAM and RCPT codes for robust image communication
}

\author{
Anam Mobin ${ }^{1}$. Ekram $\mathrm{Khan}^{2} \cdot$ Mirza Salim Beg $^{2}$
}

Received: 3 August 2020 / Accepted: 21 December 2020 / Published online: 11 January 2021

(C) The Author(s) 2021 OPEN

\begin{abstract}
In unequal error protection (UEP) schemes, the bits are protected according to their significance in terms of distortion reduction capability during the reconstruction process. The conventional UEP schemes are primarily designed either using forward error correction at application layer of network architecture, or hierarchical modulation at the physical layer. In this paper, an optimized cross-layer UEP scheme for set partitioning in hierarchical trees coded images is investigated. The proposed scheme uses rate compatible punctured turbo codes at application layer and two-layered 64-hierarchical QAM (64-HQAM) at physical layer. Simulation results show that proposed cross-layer UEP performs slightly better than application layer's optimized turbo coding for additive white Gaussian noise channel but performs significantly better in flat Rayleigh fading channel, specifically under poor channel conditions. The proposed scheme is highly bandwidth efficient having the robustness performance close to that of the scheme using hierarchical 4-PAM (H 4-PAM). Complexity analysis of the proposed solution has been carried out.
\end{abstract}

Keywords Set partitioning in hierarchical trees (SPIHT) - Unequal error protection (UEP) · Hierarchical quadrature amplitude modulation (HQAM) · Forward error correction (FEC) - Rate compatible punctured turbo (RCPT) codes . Cross-layer

\section{Introduction}

In progressive image coding, the quality of reconstructed image improves progressively with every additional bit received. The set partitioning in hierarchical trees (SPIHT) [1] is one of the most successful progressive image coding algorithms. It generates an embedded bit-stream in which bits have varying degree of significance in the sense that they contribute differently in distortion reduction during the process of image reconstruction. The bits coded earlier are more important and more vulnerable to channel errors as compared to subsequently coded bits. Thus, in order to improve the robustness against the channel errors, these bits should be protected according to their significance and vulnerability to the channel errors. The unequal error protection (UEP) schemes mainly rely on providing adequate protection to bits according to their importance to ensure that images of acceptable quality are received even under extreme channel conditions [2].

Depending upon the application specific constraints, a UEP scheme can be designed for different network layers. Among these, hierarchical modulation-based UEP at physical layer, forward error correction (FEC)-based UEP at application layer are very common. Physical layer UEP using hierarchical modulation is used in various digital video broadcast (DVB) systems and standards to simultaneously support both the high definition TV (HDTV) and standard definition TV (SDTV) services, without requiring

\footnotetext{
$\triangle$ Anam Mobin, anammobin92@gmail.com; Ekram Khan, ekhan.el@amu.ac.in; Mirza Salim Beg, salim.beg@amu.ac.in |'Department of Electronics and Communication Engineering, Jamia Millia Islamia, New Delhi, India. ${ }^{2}$ Department of Electronics Engineering,

Z. H. College of Engineering and Technology, Aligarh Muslim University, Aligarh, UP, India.
} 
any additional bandwidth [2]. Among various hierarchical modulation schemes, hierarchical quadrature amplitude modulation (HQAM) is the most commonly used and is also used in DVB applications. The utilization of HQAM as a technique to provide UEP has been extensively reported in literature. In [3, 4], 64-HQAM is used to provide UEP for data partitioned H.264 coded video. In [5] a multilevel UEP system using multiplexed HQAM is proposed for progressive image transmission. In [6] optimized 2-layered UEP scheme is proposed for wavelet coded scalable video using adaptive 16-HQAM. In [7], 2-layered and 3-layered UEP of SPIHT coded images using 64-HQAM is proposed. In [8], two-level and three-level 64-HQAM based UEP schemes are investigated for the client-driven selective streaming system. The application layer UEP can be provided using channel codes such as rate compatible punctured convolutional (RCPC) codes, reed solomon (RS) codes, turbo codes, low-density parity-check (LDPC) codes, polar codes, fountain codes etc. In [9], a UEP scheme to protect SPIHT coded images using RCPC codes is proposed and tested for noisy binary symmetric channel. In [10], a UEP scheme using RCPC codes is suggested for embedded source bit streams transmitted over lossy channels considering fixed packet size transmission. In [11], an optimized UEP scheme is proposed for SPIHT coded images using product codes that consist of RS codes and turbo codes. In [12], UEP for progressive image transmission using heuristically optimized rate compatible punctured turbo (RCPT) codes is considered. In [13], an adaptive concatenated coding scheme is proposed with turbo-like iterative decoding for scalable transmission of JPEG 2000 images. In [14], LDPC codes that are suitable for UEP are constructed. In [15], information symbols are protected unequally using fountain codes, whereas in [16] an efficient scheme is proposed to construct fountain codes with UEP property. In [17], a UEP scheme based on rateless spinal code is proposed. In [18], a rateless coding based UEP scheme is investigated by concatenating the systematic polar code (SPC) and spinal code.

In FEC based UEP, error resiliency is achieved at the cost of extra bandwidth requirement. On the other hand, HQAM based UEP is bandwidth efficient but protects high priority bits more than the low priority bits. The protection of low priority bits can be increased by increasing the average transmitted power. Therefore, for efficient utilization of communication resources such as channel bandwidth and transmitted power, a number of cross-layer UEP schemes have been proposed. The idea behind the cross-layer UEP is to exploit the advantages of the UEP techniques at different layers of network architecture and to jointly optimize them by allowing interaction among the network layers. Thus, instead of designing UEP at individual layers independently, the communication resources can be utilized more efficiently by cross-layer UEP in order to provide the best possible image quality under the limited bandwidth and transmitter power constraints.

Turbo code and 64-HQAM are combined to provide UEP to H.264 layered coded video in [19], but the error control parameters used are non-optimal. In [20], non-uniform error protection of $\mathrm{H} .263$ + layered video using RCPC code and hierarchical PSK is suggested. In [21, 22], a packetization technique combined with hierarchical modulation and RCPC coding is proposed for SPIHT coded images. In [23], a cross-layer UEP scheme for SPIHT coded scalable video is proposed in which 16-HQAM at physical layer and RS coding at application layer are combined. In [24], interlayer FEC is combined with 16-HQAM for transmission of stereoscopic 3D video. In [25], an optimized cross-layer scheme with expanding window fountain (EWF) coding at application layer and hierarchical QPSK (H-QPSK) at physical layer is investigated for scalable video transmission over DVB-handheld (DVB-H) networks. In [26], a UEP technique based on RCPC codes and hierarchical PSK modulation is presented for JPEG 2000 and H.265 standards.

The use of turbo codes in this work is motivated by the fact that it is used in a number of wireless systems and standards like WCDMA, DVB, WiMAX and LTE systems $[27,28]$. With a sufficiently large interleaver and iterative decoding, its performance approaches Shannon limit [29]. However, the large interleaver length of turbo codes increases the decoding delay and the computational complexity. The decoding delay is bearable in most of the data communication systems and the added computational complexity is counter-balanced by the high coding gain achieved by turbo codes [29]. The concept of ratecompatible puncturing primarily developed for convolutional codes, has been extended to turbo codes also. The rate-compatible punctured turbo (RCPT) codes, in which high-rate codes are embedded into the lower rate codes, are used to realize adaptive error control techniques and hybrid ARQ schemes [28].

In most of the previous works, the performance of hierarchical modulation based UEP was investigated for lower modulation levels such as hierarchical 4-PAM and 16-HQAM [6, 21-23] that are less bandwidth efficient and have the advantage of higher error resiliency. The performance of spectrally efficient 64-HQAM was investigated heuristically with non-optimal values of error control parameters $[3,4,8,19]$. In [7], the performance of 64-HQAM is investigated with optimal parameters for SPIHT coded images that fails to provide acceptable image quality under poor channel conditions.

In this paper, therefore, a higher level hierarchical modulation (64-HQAM) is combined with forward error correction (FEC) with optimal error control parameters to increase effective transmission rate while maintaining 
adequate reception quality even under poor channel conditions. A cross-layer UEP scheme is suggested in which 2-layered 64-HQAM based UEP at physical layer and RCPT codes based UEP at application layer are jointly optimized for SPIHT coded images transmitted over an additive white Gaussian noise (AWGN) channel as well as over a flat Rayleigh fading channel. In a wireless channel, the channel condition changes randomly with time, and hence the error control parameters need to be determined every time the channel condition changes and for every input image. Therefore, in order to reduce the computational complexity, an off-line optimization of error control parameters is used in this work so as to avoid the computation of optimized parameters, each time the channel condition changes. A look-up table is designed that stores optimized parameters for wide range of channel conditions. This approach greatly reduces the computational complexity and delay associated with real-time optimization of parameters, and hence makes the technique suitable for real-time image communication.

This paper is organized as follows. In Sect. 2, UEP schemes used at individual layers of communication network are reviewed. Also, partitioning of SPIHT coded bit stream to protect the bits according to their sensitivity is presented in Sect. 2. The proposed cross-layer UEP scheme that exploits the error control techniques of two different layers is presented in Sect. 3. Simulation results and performance analysis are presented in Sect. 4. Finally, the paper is concluded in Sect. 5.

\section{UEP at individual layers}

In this section, the conventional UEP schemes used at different network layers, namely hierachical modulation (64-HQAM) at physical layer and FEC (RCPT codes) at application layer are discussed. In order to apply required protection to the bits in UEP schemes, the bitstreams need to be prioritized (partitioned according to their importance). Therefore, this section will begin with the data partitioning strategy that can be used in single layer UEP schemes as well as proposed cross-layer UEP scheme.

\subsection{Data partitioning}

In order to protect the bits according to their vulnerability to channel errors and to exploit the advantages of UEP schemes, the data (bit-stream) need to be partitioned efficiently into different priority layers according to their importance. SPIHT [1] being a bit-plane based coder, generates an embedded bit-stream, in which early generated bits are of higher significance than the later coded bits. SPIHT coder performs two passes in each bit-plane, namely sorting $(\mathrm{S})$ and refinement $(\mathrm{R})$ passes. In sorting passes of higher bit-planes, more important and more error sensitive bits are generated as compared to that generated in lower bit-plane sorting passes and all refinement passes. In this work, SPIHT coded bit-stream is divided into two parts as shown in Fig. 1. Sorting bits are extracted from the coded bit-stream and grouped together to form the first part, whereas all the refinement bits form the second part. Also, bits generated in a higher bit-plane pass are placed earlier than those generated in a lower bit-plane pass. Thus, the partitioning scheme arranges bits in decreasing order of their significance and error sensitivity. Therefore, the bit-stream can be split into two priority layers: high priority (HP) layer that constitutes 33\% of entire bit-stream, and low priority (LP) layer that constitutes $67 \%$ of entire bit-stream, to provide UEP, as illustrated in Fig. 1.

The bits partitioned into different priority layers can be protected according to their error sensitivity by either using hierarchical modulation or error control coding such as RCPT codes. These two schemes are briefly discussed below.

\subsection{UEP at physical layer using 64-HQAM}

UEP at physical layer is provided using HQAM by mapping more significant information, High Priority (HP) bits of Fig. 1, to more error resilient pair of bits of HQAM symbol and less significant information i.e. Low Priority (LP) bits to more vulnerable pair of bits of HQAM symbol. Figure 2 shows the constellation diagram of 2-layered 64-HQAM, which requires 6 bits/symbol. The two most significant bits (MSBs) of the signal points are occupied by HP data bits and the remaining 4 bits by LP data bits. The use of gray coding ensures that points within a quadrant have the same HP bits. Then data is protected unequally by adjusting the modulation parameter, $a$, as defined in Eq. (1).

$\alpha=\mathrm{a} / \mathrm{b}$

When $a=1$, the constellation is same as that of conventional/non-hierarchical 64-QAM. When $a>1$, while the

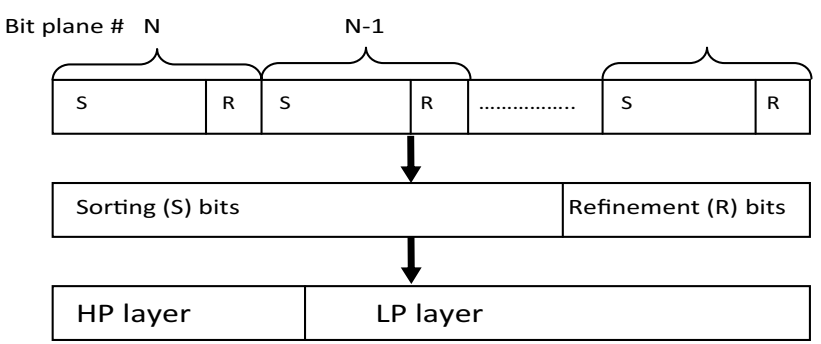

Fig. 1 Partitioning of SPIHT bit-stream into 2-layers 


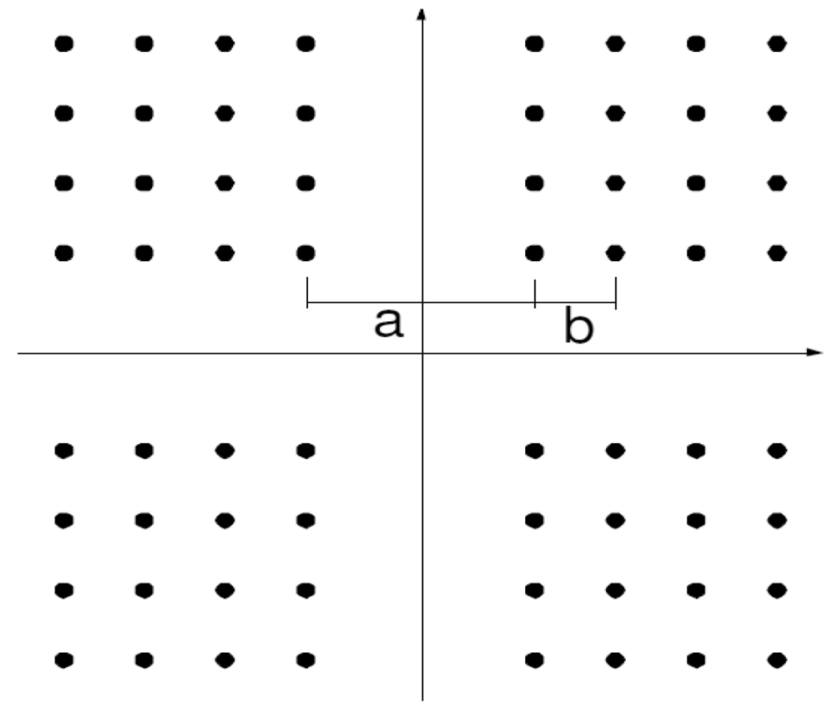

Fig. 2 Constellation diagram of 2-layered 64-HQAM

average symbol energy is kept constant, then HP bits are protected more strongly than LP bits, That is, the higher error resiliency of HP bits is achieved at the cost of reduced noise immunity to LP bits.

\subsection{UEP at application layer using RCPT codes}

In FEC based UEP scheme used at application layer, the high priority data is protected with a lower rate channel code (stronger channel code) and the low priority data with a higher rate channel code (weaker channel code). The RCPC codes, turbo codes, RS codes, fountain codes are widely used to provide UEP. Since, we have used RCPT codes, which is a variant of turbo codes, we will first study and analyze the performance of turbo codes in comparison to other codes such as RS codes. A basic turbo encoder is a recursive systematic encoder that utilizes two recursive systematic convolutional encoders connected in parallel, and information bits are passed through second encoder after reordering them using a certain permutation algorithm [29]. Figure 3 compares the bit error rate (BER) performance of RS and turbo codes with 64-QAM in AWGN channel. It can be observed that turbo code performs significantly better than RS code for a given value of channel coding rate (say $r=1 / 3$ ), and this is the main motivation to use turbo codes in this work.

The use of turbo codes in a UEP scheme requires encoding of different priority layers with turbo codes of different coding rates. Instead, a better option is to use a single turbo code that can provide variable code rate. Such a variable rate turbo coding is accomplished by puncturing the parity check bits at the output of the two convolutional encoders (that are parts of a basic turbo encoder)

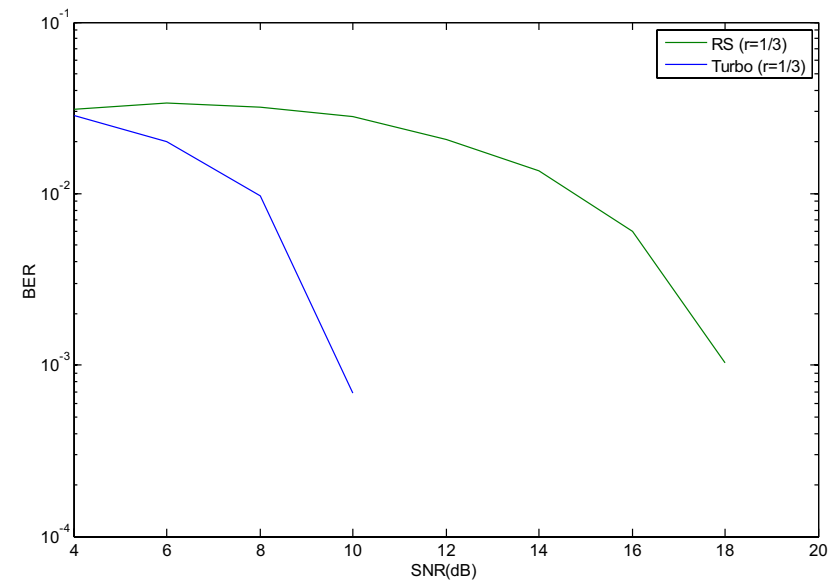

Fig. 3 BER performance comparison of Turbo and RS codes for coding rate, $r=1 / 3$ with 64-QAM in AWGN channel

by different amounts. With puncturing the same decoder can be used at the receiver side irrespective of the coding rate. Thus, making the system highly flexible without any significant increase in system complexity. The selected puncturing matrices are required to satisfy a rate compatibility criterion such that the codes with lower code rate/ with higher redundancy output the same coded bits as generated by all the codes with higher code rates besides some extra redundant bits. The resulting higher rate codes obtained by puncturing a single low rate turbo code are called the rate-compatible punctured turbo (RCPT) codes.

Let $1 / n$ be the parent code rate and $P$ be the puncturing period, corresponding to $\mathrm{P}$ input information bits. Hence, a total of $\mathrm{nP}$ coded bits are transmitted in one period. A puncturing matrix $M$ associated with the coded output bits is defined as

$M=\left[\begin{array}{llll}P_{11} & P_{12} & \ldots & P_{1 p} \\ P_{21} & P_{22} & \ldots & P_{2 p} \\ \vdots & \vdots & \vdots & \vdots \\ P_{n 1} & P_{n 2} & \ldots & P_{n p}\end{array}\right]$

where each column of $M$ represents the $n$ coded bits for each input information bit. When $P_{i j}=1$, the output bit of the encoder corresponding to that matrix element is transmitted. When $\mathrm{P}_{\mathrm{ij}}=0$, the output bit of the encoder corresponding to that matrix element is deleted. Thus, the puncturing period $\mathrm{P}$ and the total number of bits punctured at the output of encoder completely determine the code rate. Good punctured codes can be designed by searching and choosing puncturing matrices that result in maximum free distance [29].

Figure 4 compares the BER performances of RCPT codes with 64-QAM in AWGN channel. It can be observed that as the channel coding rate, ' $r$ ' increases, the BER increases. 
Fig. 4 BER performances of RCPT codes with 64-QAM in AWGN channel

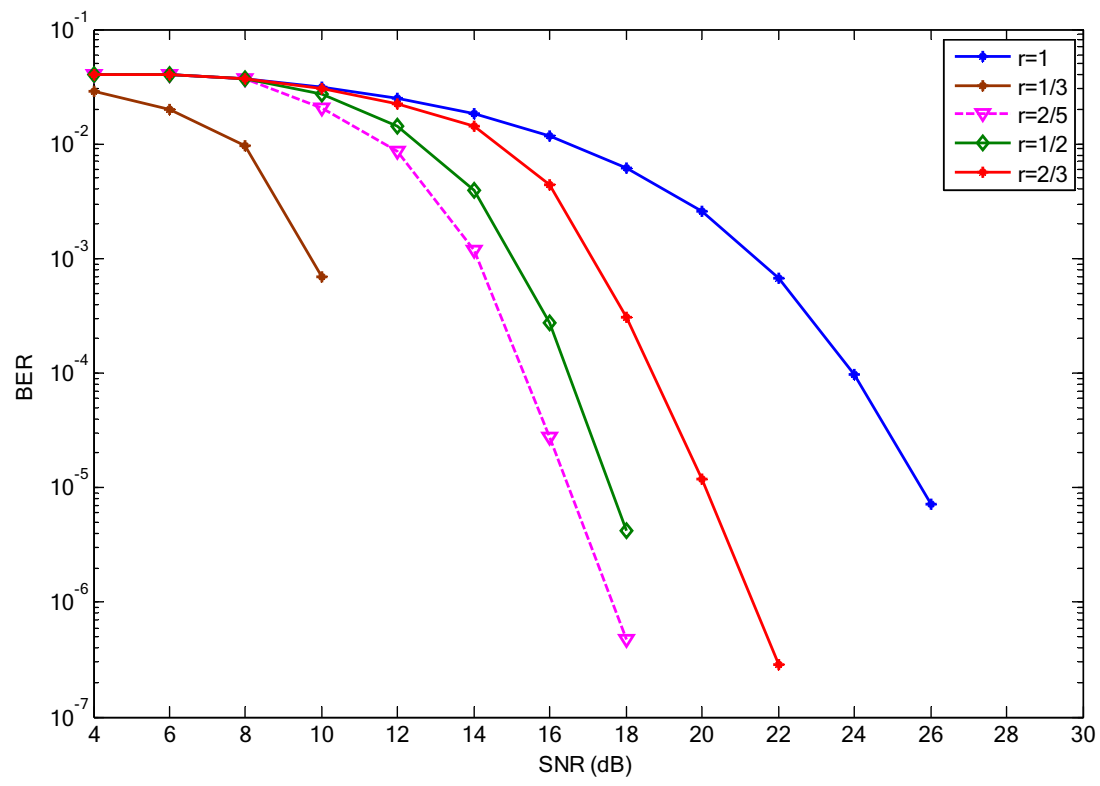

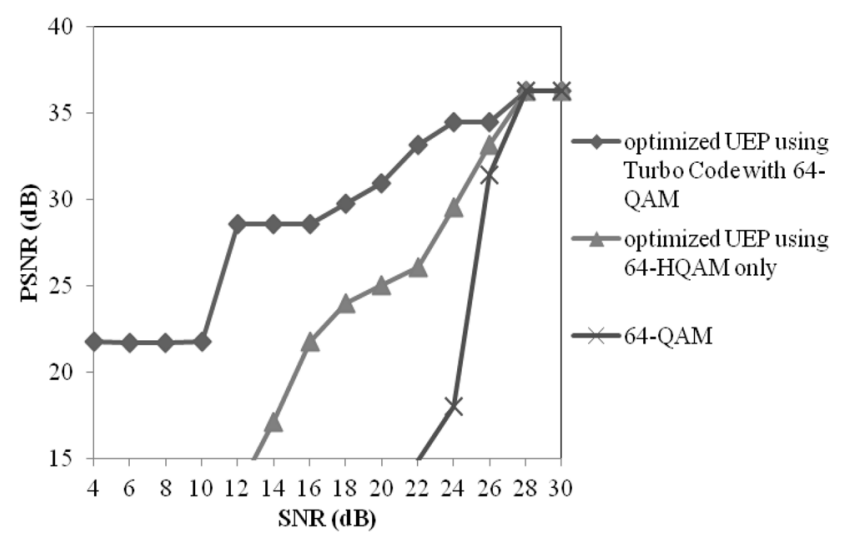

Fig. 5 Performance comparison of single-layer UEP schemes for Barbara image

This is due to the reduced protection provided to the source bits by puncturing some of the parity bits. It can also be observed that with channel coding rate, $r=1 / 3$, at signal to noise ratio $(\mathrm{SNR})=10 \mathrm{~dB}$, the same $B E R$ is achieved as that with no channel coding at $S N R=22 \mathrm{~dB}$, thus providing a coding gain of about $12 \mathrm{~dB}$.

In order to verify the robustness performance of single-layer UEP, Fig. 5 compares the performance of various single layer UEP schemes for standard $512 \times 512$ grayscale image Barbara, in terms of peak signal to noise ratio (PSNR) of reconstructed image. It can be observed that RCPT code based UEP at application layer significantly outperforms 64-HQAM based UEP at physical layer. However, the quality of received image for even RCPT code based UEP under poor channel conditions (for SNR in the range of 4-10 dB) is unacceptable (as PSNR is less than $25 \mathrm{~dB}$ ). Therefore, there is need to improve the quality of received images, specially, under poor channel conditions, for which we propose a cross-layer UEP scheme that exploits the benefits of both FEC and HQAM to receive the best possible image quality under limited communication resources.

\section{Proposed cross-layer UEP scheme}

In single layer UEP schemes (discussed in the previous section) there exists a tradeoff between error resiliency and communication resource requirement. As stated earlier, FEC based UEP achieves error resiliency at the cost of extra bandwidth requirement, whereas in HQAM based UEP, high priority bits are protected more than the low priority bits, thereby increasing the bit error rate (which may be compensated by increasing the average transmission power). To overcome the limitations of these single layer UEP schemes, a cross-layer UEP scheme is proposed in this paper. The proposed UEP scheme, combines HQAM at physical layer with FEC at application layer to enhance the error resiliency of LP bits without the need to increase the transmit power. Also, using a higher rate (weaker) channel code in conjunction with HQAM would provide sufficient protection to HP bits thus, saving the bandwidth. The main idea behind the cross-layer scheme is to allow interaction among the layers, for efficient utilization of available resources, such as bandwidth and transmitted power.

The block diagram of the proposed image communication system with a cross-layer UEP scheme is shown in Fig. 6 . The proposed system assumes that the channel conditions are known at the transmitter side. This is a fair 
Fig. 6 Proposed image communication system with crosslayer UEP

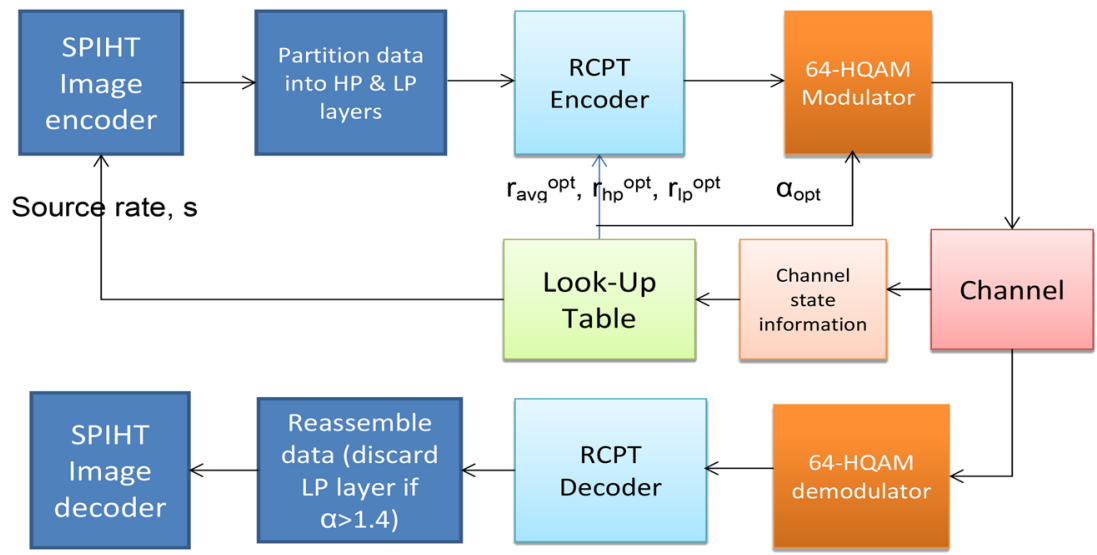

assumption in a packet-based network, where current channel state information (channel SNR) may be included in acknowledgment packets. In order to minimize the usage of communication resources, proposed system uses a pre-designed look-up table that contains optimal values of error control parameters for set of possible channel conditions. The use of look-up table in the proposed system reduces its complexity. The system works as follows. The bit-stream of SPIHT coded image is first partitioned in two priority levels, namely HP and LP sub-streams (as depicted in Fig. 1). These HP and LP sub-streams are then RCPT coded using optimized coding rates $\mathrm{r}_{\mathrm{hp}}^{\mathrm{opt}}$ and $\mathrm{r}_{\mathrm{lp}}^{\mathrm{opt}}$ respectively. The optimal values of code rates are to be searched from a pre-designed look-up table (design of look-up table is discussed later in this section). RCPT coded sub-streams are then mapped on the 64-HQAM with optimal modulation parameter, $a_{\text {opt }}$ again from the look-up table (LUT) for given channel conditions. The HQAM modulated signal is then transmitted over the erroneous channel. At the receiver side, reverse process is performed to reconstruct the image. Moreover, the optimal modulation parameter, mother code parameters, and puncturing patterns are placed in the packet header and transmitted, to facilitate demodulation and decoding performed at the receiver. It is assumed that the header is protected heavily with strong channel coding and received with no errors. It is observed that if the value of $a_{\text {opt }}$ is high then LP layer is received with large number of errors [7], thus degrading the restored image quality. Hence, if the value of $a_{o p t}$ at the receiver is found to be greater than a threshold value (which is 1.4), LP bits are discarded and not used in the image reconstruction.

As mentioned earlier, instead of finding the optimal values of error control parameters every time the channel condition changes, a look-up table based approach is used in the proposed system. The optimal values of error control parameters are obtained by taking average of the optimal values obtained for different test images that maximize PSNR of reconstructed image. The other quality parameters such as structural similarity index measure (SSIM) [30] may also be used as a metric for optimization. The average optimized error control parameters are then stored in a look-up table for a set of possible SNRs. A separate look-up table may be designed for each particular class of test images and is therefore suitable for that particular application. Though the initial cost of designing the look-up table is high but since it has to be designed only once in the beginning before the system starts operating, it will not affect the operational complexity of the system. Therefore, the operational complexity of the proposed system is minimal as it requires only searching of optimal error control parameters from the pre-designed look-up table and is therefore suitable for real-time communication.

For UEP, HP and LP bits are coded with channel coding rate $r_{h p}$ and $r_{l p}$ respectively. Let, $r_{a v g}$ denote the average coding rate of the entire source coded bit-stream. Due to the limitation of two-layered 64-HQAM constellation that offers a capacity of $33.33 \%$ and $66.66 \%$ to HP and LP bits respectively, the percentage of HP and LP channel coded bits are kept constant in this work, at $33.33 \%$ and $66.66 \%$ respectively, of the total number of channel coded bits (ch) which is also a constant. This is done because the aim of this work is to combine two-layered 64-HQAM with RCPT codes. Hence, the value of $r_{h p}$ and $r_{\text {lp }}$ dictates the length of HP source bit-stream $\left(s_{h p}\right)$ and LP source bit-stream ( $\left.s_{\mid p}\right)$ respectively, as shown in Eqs. (2) and (3):

$s_{h p}=0.33 \times c h \times r_{h p}$

$s_{l p}=0.66 \times c h \times r_{l p}$

Let $\mathrm{ch}_{\mathrm{hp}}$ and $\mathrm{ch}_{\mathrm{Ip}}$ denote the length of HP and LP channel coded bit-streams respectively. Then, the average channel code rate, $r_{\text {avg }}$ can be obtained from Eq. (4). 
$r_{a v g}=\frac{s_{h p}+s_{l p}}{c h_{h p}+c h_{l p}}$

Since, HP and LP channel coded bits are kept fixed at $33.33 \%$ and $66.66 \%$ of the total channel coded bits respectively. Hence,

$c h_{l p}=2 c h_{h p}$

Moreover

$r_{h p}=\frac{s_{h p}}{c h_{h p}}$

$r_{l p}=\frac{s_{l p}}{c h_{l p}}$

Using Eqs. (5)-(7), Eq. (4) can also be written as

$r_{a v g}=\frac{c h_{h p} \times r_{h p}+2 c h_{h p} \times r_{l p}}{c h_{h p}+2 c h_{h p}}$

Simplifying Eq. (8), we get

$r_{\text {avg }}=\frac{r_{h p}+2 r_{l p}}{3}$

Equation (9) is used to calculate $r_{a v g}$ in this work. Depending upon the channel state information (CSI), the optimal error control parameters $\left\{r_{\mathrm{avg}}^{\mathrm{opt}} \mathrm{r}_{\mathrm{hp}}^{\mathrm{opt}}, \mathrm{r}_{\mathrm{lp}}^{\mathrm{opt}}, \mathrm{a}_{\mathrm{opt}}, \mathrm{s}\right\}$ need to be searched from a look up table that stores optimal values of parameters for different SNR values (that specify the channel condition) and provide these parameters to source, and channel coding blocks at the application layer and modulation block at the physical layer. Here, s denotes the total length of source-coded bit-stream. Perfect CSI is assumed at receiver. The CSI must be coded with heavy protection and fed back to the transmitter via a reliable feedback channel. It is assumed that CSI is received without any error.

\section{Simulation results}

The performance of proposed cross-layer UEP scheme was tested for standard ( 8 bpp) $512 \times 512$ grayscale images, coded using SPIHT algorithm at the rate of $1 \mathrm{bpp}$. To construct the look-up table, a set of $10,512 \times 512$ grayscale images (Lena, Barbara, Goldhill, Peppers, Man, Couple, Baboon, Boat, Airplane and House) was considered. The proposed scheme was simulated in MATLAB8.3 (R2014a). Systematic Rate Compatible Puncture Turbo (S-RCPT) Codes (no systematic/information bits are punctured) with parent code rate of $1 / 3$, generator $(13,15)_{\text {oct }}$ and constraint length $=4$ are used. The RCPT codes with a set of code rates $\{1 / 3,2 / 5,1 / 2,4 / 7,2 / 3,4 / 5\}$ are obtained using puncturing matrices shown in Table 1 [28]. An iterative MAP decoding with five decoding iterations is used. Two channel models, namely AWGN and flat Rayleigh fading channel were considered. The flat fading channel with Doppler spread of $80 \mathrm{~Hz}$ (representing moderate speed vehicular traffic), carrier frequency of $900 \mathrm{MHz}$ (representing a commonly used band for mobile phones) and a moderate symbol transmission rate of 4800 symbols per second was considered. For comparison purpose, RS codes with following parameters were used: the number of bits per symbol $(m)=8$, the total number of symbols in a codeword $(n)=2^{m}-1$ and primitive polynomial: $D^{8}+D+1$.

The optimized values of error control parameters for each tested SNR for proposed cross-layer UEP scheme are stored in the look up tables. These are shown in Tables 2 and 3 for AWGN channel and for flat Rayleigh fading channel respectively. From Table 2, it can be inferred that in AWGN channel, at low SNR values the cross-layer UEP gives optimal protection but at high SNR values equal error protection (EEP) using RCPT codes gives optimal protection. For flat Rayleigh fading channel, the cross-layer UEP gives optimal protection in the lower SNR range (poor channel conditions) and also at some high SNR values. This shows that the performance of RCPT codes in fading channel is not as good as they perform in AWGN channel [19].

Figure 7 compares the performance of proposed crosslayer UEP with that of single-layered UEP schemes in terms of objective quality of reconstructed images against various AWGN channel conditions (i.e. PSNR vs SNR) for four standard images namely, Lena, Goldhill, Peppers, and Barbara. From these figures, it can be observed that optimized cross-layer UEP has a performance gain of about
Table 1 Rate-compatible punctured turbo codes constructed from rate $1 / 3$ with puncturing period, $P=8[28]$

\begin{tabular}{|c|c|}
\hline Rate & Puncturing matrix \\
\hline $1 / 3$ & $\begin{array}{llllllll}1 & 1 & 1 & 1 & 1 & 1 & 1 & 1 \\
1 & 1 & 1 & 1 & 1 & 1 & 1 & 1 \\
1 & 1 & 1 & 1 & 1 & 1 & 1 & 1\end{array}$ \\
\hline $2 / 5$ & $\begin{array}{llllllll}1 & 1 & 1 & 1 & 1 & 1 & 1 & 1 \\
1 & 1 & 0 & 1 & 1 & 1 & 1 & 1 \\
0 & 1 & 1 & 1 & 0 & 1 & 1 & 0\end{array}$ \\
\hline $1 / 2$ & $\begin{array}{llllllll}1 & 1 & 1 & 1 & 1 & 1 & 1 & 1 \\
0 & 0 & 0 & 0 & 1 & 1 & 1 & 1 \\
0 & 0 & 1 & 1 & 0 & 1 & 1 & 0\end{array}$ \\
\hline $4 / 7$ & $\begin{array}{llllllll}1 & 1 & 1 & 1 & 1 & 1 & 1 & 1 \\
0 & 0 & 0 & 0 & 1 & 1 & 0 & 1 \\
0 & 0 & 1 & 1 & 0 & 1 & 0 & 0\end{array}$ \\
\hline $2 / 3$ & $\begin{array}{llllllll}1 & 1 & 1 & 1 & 1 & 1 & 1 & 1 \\
0 & 0 & 0 & 0 & 0 & 1 & 0 & 1 \\
0 & 0 & 1 & 0 & 0 & 1 & 0 & 0\end{array}$ \\
\hline $4 / 5$ & $\begin{array}{llllllll}1 & 1 & 1 & 1 & 1 & 1 & 1 & 1 \\
0 & 0 & 0 & 0 & 0 & 1 & 0 & 0 \\
0 & 0 & 1 & 0 & 0 & 0 & 0 & 0\end{array}$ \\
\hline
\end{tabular}

SN Applied Sciences 
Table 2 Look up table of optimal error control parameters for proposed cross-layer scheme in AWGN channel

\begin{tabular}{lllll}
\hline SNR (dB) & $\mathrm{r}_{\mathrm{hp}}^{\mathrm{opt}}$ & $\mathrm{r}_{\mathrm{lp}}^{\mathrm{opt}}$ & $\mathrm{r}_{\mathrm{avg}}^{\mathrm{opt}}$ & $\mathrm{a}_{\mathrm{opt}}$ \\
\hline 4 & $1 / 3$ & $2 / 5$ & 0.377 & 4.6 \\
6 & $2 / 5$ & $1 / 2$ & 0.47 & 7.2 \\
8 & $1 / 2$ & $4 / 7$ & 0.547 & 7.3 \\
10 & $4 / 7$ & $2 / 3$ & 0.635 & 4.3 \\
12 & $1 / 3$ & $1 / 3$ & 0.33 & 1 \\
14 & $1 / 3$ & $1 / 3$ & 0.33 & 1 \\
16 & $1 / 3$ & $1 / 3$ & 0.33 & 1 \\
18 & $2 / 5$ & $2 / 5$ & 0.4 & 1 \\
20 & $4 / 7$ & $4 / 7$ & 0.57 & 1 \\
22 & $2 / 3$ & $2 / 3$ & 0.66 & 1 \\
24 & $4 / 5$ & $4 / 5$ & 0.8 & 1 \\
26 & $4 / 5$ & $4 / 5$ & 0.8 & 1 \\
28 & 1 & 1 & 1 & 1 \\
30 & 1 & 1 & 1 & 1 \\
\hline
\end{tabular}

Table 3 Look up table of optimal error control parameters for proposed cross-layer scheme in flat Rayleigh fading channel

\begin{tabular}{|c|c|c|c|c|}
\hline SNR (dB) & $\mathrm{r}_{\mathrm{hp}}^{\mathrm{opt}}$ & $r_{l p}^{o p t}$ & $\mathrm{r}_{\mathrm{avg}}^{\mathrm{opt}}$ & $a_{o p t}$ \\
\hline 14 & $1 / 3$ & $2 / 5$ & 0.377 & 6 \\
\hline 16 & $1 / 3$ & $2 / 5$ & 0.377 & 5.6 \\
\hline 18 & $1 / 3$ & $2 / 5$ & 0.377 & 4.7 \\
\hline 20 & $1 / 3$ & $2 / 5$ & 0.377 & 3.9 \\
\hline 22 & $1 / 3$ & $2 / 5$ & 0.377 & 3 \\
\hline 24 & $1 / 3$ & $2 / 5$ & 0.377 & 2.6 \\
\hline 26 & $1 / 3$ & $2 / 5$ & 0.377 & 2 \\
\hline 28 & $1 / 3$ & $1 / 3$ & 0.33 & 1.4 \\
\hline 30 & $1 / 3$ & $1 / 3$ & 0.33 & 1.3 \\
\hline 32 & $1 / 3$ & $1 / 3$ & 0.33 & 1 \\
\hline 34 & $1 / 3$ & $1 / 3$ & 0.33 & 1 \\
\hline 36 & $1 / 3$ & $1 / 3$ & 0.33 & 1 \\
\hline 38 & $1 / 3$ & $1 / 3$ & 0.33 & 1 \\
\hline 40 & $1 / 3$ & $1 / 3$ & 0.33 & 1 \\
\hline 42 & $1 / 3$ & $1 / 3$ & 0.33 & 1 \\
\hline 44 & $1 / 3$ & $1 / 3$ & 0.33 & 1 \\
\hline 46 & $2 / 5$ & $2 / 5$ & 0.4 & 1.2 \\
\hline 48 & $2 / 5$ & $2 / 5$ & 0.4 & 1 \\
\hline 50 & $4 / 7$ & $4 / 7$ & 0.57 & 1 \\
\hline 52 & $4 / 7$ & $4 / 7$ & 0.57 & 1 \\
\hline 54 & $2 / 3$ & $2 / 3$ & 0.66 & 1.2 \\
\hline 56 & $2 / 3$ & $2 / 3$ & 0.66 & 1 \\
\hline 58 & $4 / 5$ & $4 / 5$ & 0.8 & 1.1 \\
\hline 60 & 1 & 1 & 1 & 1.3 \\
\hline 62 & 1 & 1 & 1 & 1 \\
\hline 64 & 1 & 1 & 1 & 1 \\
\hline
\end{tabular}

2-7 dB in the poor channel conditions (in the SNR range of 4-10 dB), where single-layer UEP has relatively poor performance. However, under good channel conditions (SNR in the range 12-30 dB), RCPT-based UEP gives optimal performance. This observation is in conformity with the optimal error control parameters obtained in the high SNR range as shown in Table 2. From Fig. 7a, it can be further observed that in AWGN channel at $S N R=10 \mathrm{~dB}$, the optimized cross-layer UEP outperforms optimized UEP using 64-HQAM, by about $21 \mathrm{~dB}$ and optimized UEP using RCPT codes, by about $6 \mathrm{~dB}$. Figure 8 compares the subjective image quality of image Lena transmitted over AWGN channel with $S N R=10 \mathrm{~dB}$, protected using different optimized UEP schemes. It can be observed that HQAM based optimized UEP results in reconstruction failure, whereas, using channel coding based optimized UEP, image quality is improved significantly. Moreover, the proposed cross-layer UEP scheme gives the image of acceptable quality. The positive values of SSIM lie in the range [0, 1] [30]. The closer the value to 1 , higher is the structural similarity between the restored image and the original image. In order to further strengthen our claims, we have also calculated SSIM values of these images, which are found to be 0.3457 , 0.6432 and 0.8620 for images shown in Fig. $8 \mathrm{c}-\mathrm{e}$ respectively. This shows that the proposed scheme improves the structural similarity between the original and reconstructed images.

Figure 9 compares the performance of various optimized UEP schemes for Lena and Goldhill images transmitted (after SPIHT coding) over flat Rayleigh fading channel. It can be observed that proposed cross-layer UEP (with optimized parameters), outperforms RCPT-based UEP by about 3-9 dB at channel SNR in the range of $14-26 \mathrm{~dB}$. From Fig. 9a it can further be observed that in flat fading Rayleigh channel at SNR $=20 \mathrm{~dB}$ optimized cross-layer UEP outperforms optimized 64-HQAM based UEP by about $10 \mathrm{~dB}$ and optimized RCPT codes based UEP, by about $6 \mathrm{~dB}$. Further, it can be seen that higher SNR is required for fading channel than AWGN channel for getting the same image quality, as expected.

Table 4 compares the performance of proposed UEP scheme to that of a state-of-the-art UEP scheme presented in [21] for Lena image transmitted over an AWGN channel. The UEP scheme developed in [21] uses the combination of hierarchical 4-PAM (H 4-PAM) and RCPC coding for SPIHT coded images. The proposed UEP scheme seems to be similar to that presented in [21] in the sense that both exploit the advantages of hierarchical modulation and FEC and are tested over a similar dataset. From, Table 4 it can be observed that the performance of the proposed scheme is almost the same as that of the scheme used in [21] in the SNR range of 4-8 dB, but it performs slightly better in the SNR range of 10-12 dB. In [21] H 4-PAM is used 


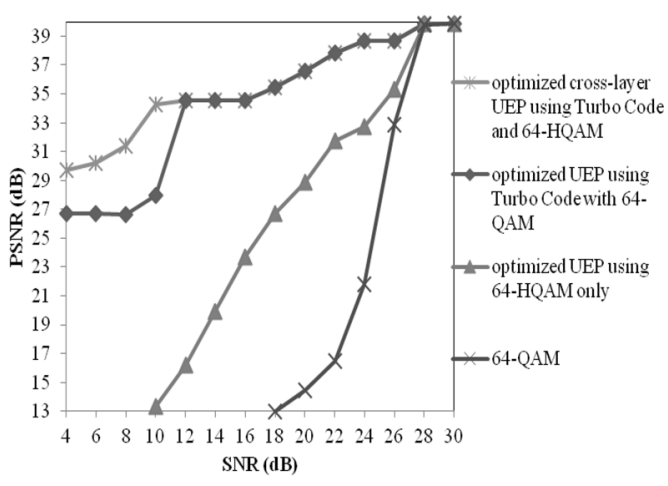

(a)

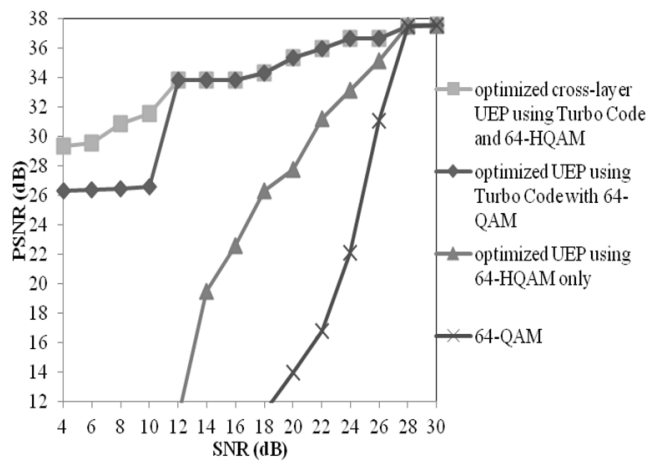

(c)

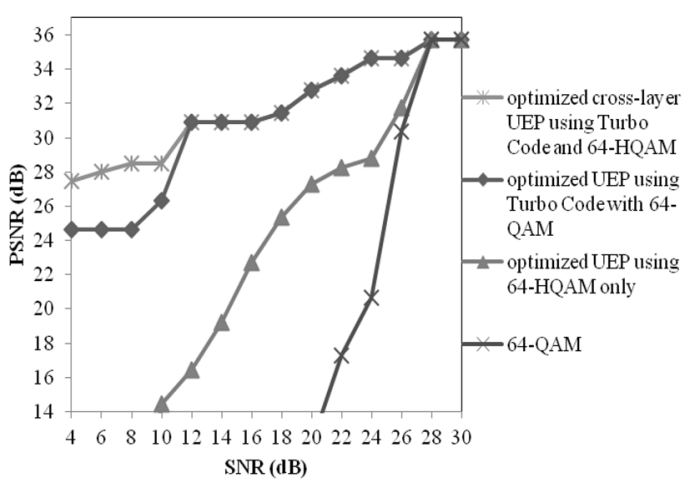

(b)

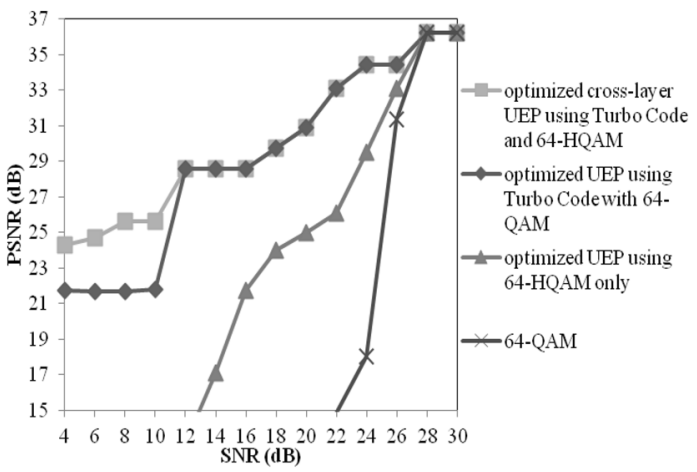

(d)

Fig. 7 Performance of optimized cross-layer UEP in AWGN channel for a Lena, b Goldhill, $\mathbf{c}$ Peppers, and $\mathbf{d}$ Barbara images

that transmits 2 bits per symbol over a given bandwidth, whereas 64-HQAM transmits 6 bits per symbol. Moreover, $\mathrm{H}$ 4-PAM has the advantage of higher resiliency to noise over 64-HQAM for same value of average symbol energy. Hence, it can be concluded that the proposed scheme is 3 times more bandwidth efficient than the scheme used in [21], with almost similar performance in terms of PSNR.

\subsection{Complexity analysis of the proposed method}

In this section the complexity analysis is performed in terms of estimated execution time. Let $t_{1}$ be the time required to encode the image and partition the coded bit stream into two priority layers. Let $t_{2}$ be the time required to perform channel coding, modulation and allocation of error control parameters. Similarly, time required to perform demodulation, channel decoding is assumed to be $t_{3}$. Let time required to reassemble the data and decode the image be $t_{4}$. Channel transmission time is assumed to be $t_{5}$. It is assumed that both transmitter and receiver have the access of test images. Also, each experiment was run 20 times and average PSNR was recorded. Therefore, total time to obtain the average PSNR of a test image, at a given SNR will be
$T=\left(t_{1}+t_{2}+t_{3}+t_{4}+t_{5}\right) \times 20$

Then optimal values of error control parameters are searched that maximize PSNR of reconstructed image by testing every possible combination of error control parameters. This requires the process to be repeated $N$ number of times, which is given by

$N=r_{\text {comb }} \times \frac{\left(\alpha_{\max }-1\right)}{\delta \alpha}$

where $r_{\text {comb }}$ is the total no. of possible combinations of $r_{h p}$ and $r_{l p}$, which can take values from the set of code rates $\{1 / 3,2 / 5,1 / 2,4 / 7,2 / 3,4 / 5\}$ such that $r_{h p} \leq r_{l p}$. Here, $r_{\text {comb }}$ is found to be 21. $\alpha_{\max }$ is the maximum value of $a$, which is set at 10 and $\delta a$ is the step size of $a$ which is set at 0.2. Then the average of optimal values of error control parameters obtained for $X$ no. of test images at different SNRs (assuming $Y$ no. of SNRs) is calculated and stored in a LUT. Therefore, the total time required to design the LUT can be estimated as

$T_{\text {total }}=X \times Y \times N \times T$ 


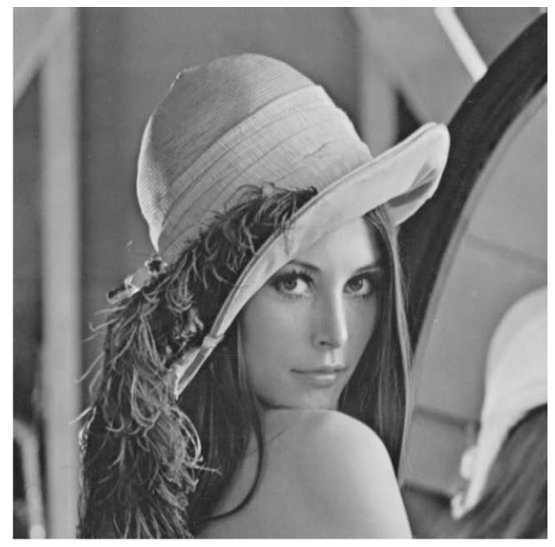

(a)

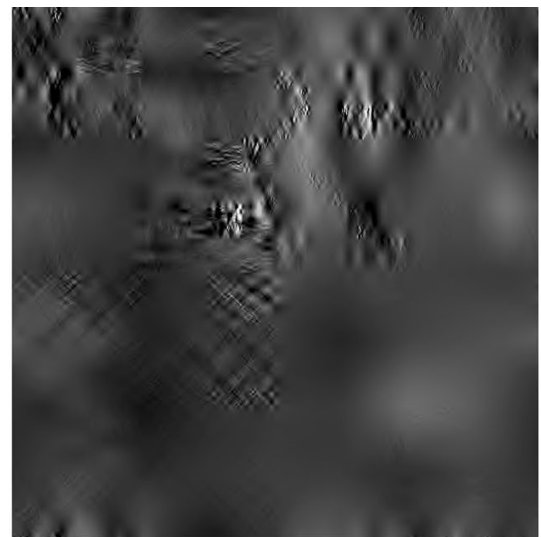

(b)

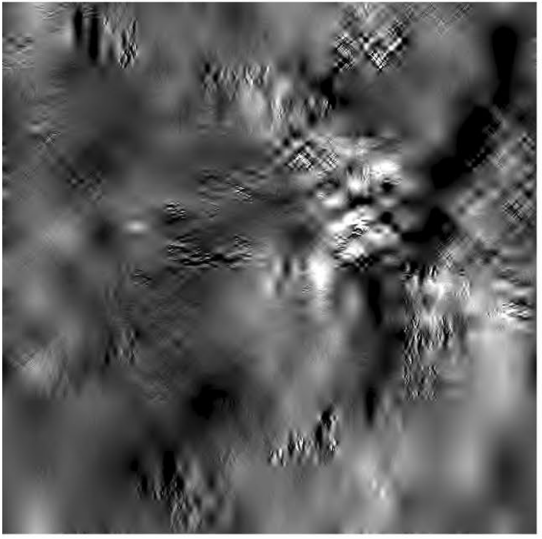

(c)

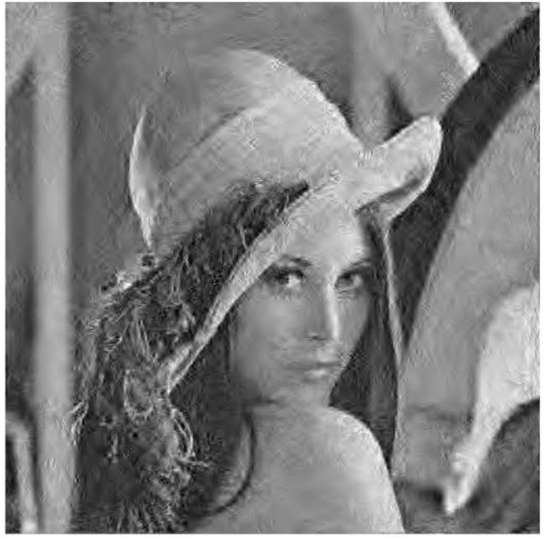

(d)

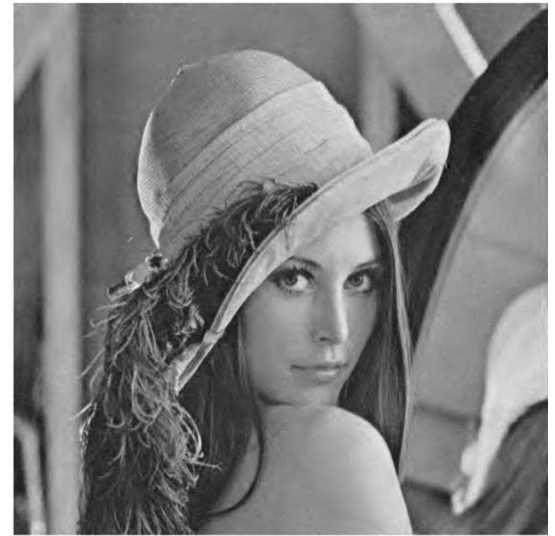

(e)

Fig. 8 Subjective quality comparison using different schemes in AWGN channel at $S N R=10 \mathrm{~dB}$. a Original image, b no protection, $\mathbf{c}$ optimized 64-HQAM, d optimized Turbo coding, and e optimized cross-layer UEP

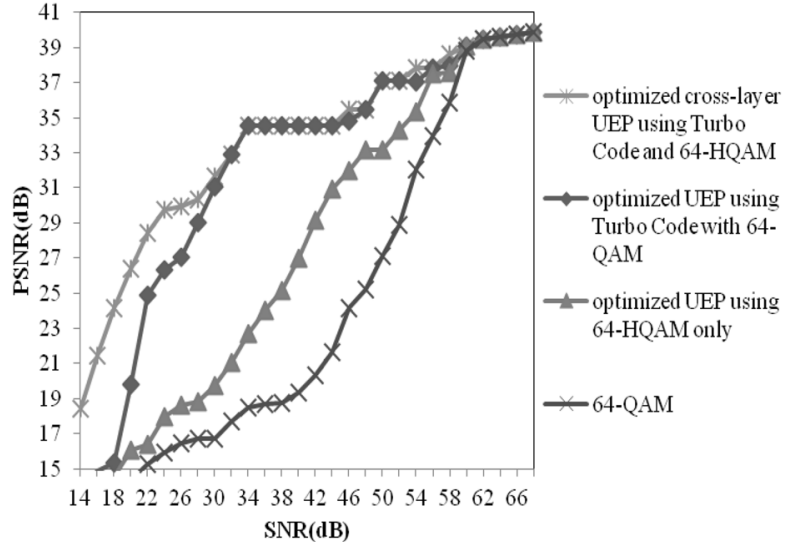

(a)

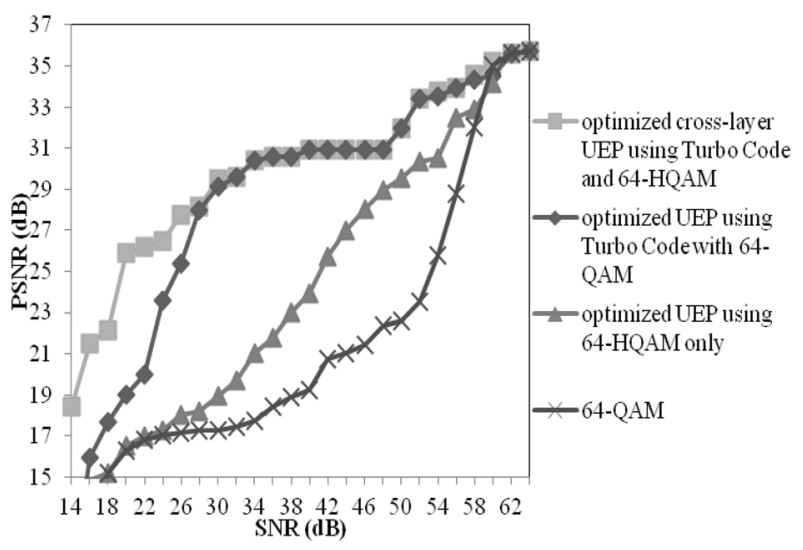

(b)

Fig. 9 Performance of optimized cross-layer UEP in flat Rayleigh fading channel for a Lena, and $\mathbf{b}$ Goldhill images

\section{SN Applied Sciences}


Table 4 Performance comparison of proposed optimized cross-layer UEP with scheme used in [21]

\begin{tabular}{lllllll}
\hline UEP scheme & SNR $(\mathrm{dB})$ & 4 & 6 & 8 & 10 & 12 \\
\hline Arslan, Cosman UEP scheme [21] & PSNR (dB) & 28.3 & 29.6 & 30.8 & 31.8 & 32.9 \\
Proposed Cross-layer UEP scheme & PSNR (dB) & 28.54 & 29.32 & 30.71 & 33.09 & 33.32 \\
\hline
\end{tabular}

Note that the LUT has to be designed only once, before the actual image communication starts. Let $t_{0}$ be the time required to search optimal error control parameters from the LUT for a specific SNR. Then time required to transmit an image using the proposed scheme, is given by

$T_{P}=\left(t_{1}+t_{2}+t_{o}\right)$

If real-time optimization is performed then the time taken to transmit an image, is given by

$T_{R}=\left(t_{1}+t_{2}+N \times T\right)$

The value of product $N \times T$ is quite large in comparison with LUT search time $t_{0}$. Hence, this approach of offline optimization greatly reduces the computational complexity and average execution time associated with real-time optimization of parameters. This in turn will also reduce the battery drainage of mobile/portable devices.

\section{Conclusions}

In this paper, a cross-layer UEP scheme has been proposed that jointly optimizes the parameters of RCPT codes and two-layered 64-HQAM, in order to achieve the best possible restored image quality using limited communication resources. It was observed that cross-layer UEP has relatively better performance better than that of single layer optimized UEP schemes, both for AWGN as well as for flat Rayleigh fading channels. The better performance is more prominent at lower SNRs (or poor channel conditions). Our investigations further reveal that at higher SNRs (under good channel conditions); the equal error protection (EEP) with optimized RCPT codes gives the best image quality. It was also observed that the proposed cross-layer scheme is highly bandwidth efficient with performance approaching that of a UEP scheme using a lower modulation level.

The proposed cross-layer UEP scheme can be extended for colored images and video. Further the scheme needs to be tested over frequency selective fading channels.

Authors' contribution Conceptualization: Ekram Khan; Methodology: Anam Mobin, Ekram Khan, and Mirza Salim Beg; Formal analysis and investigation: Anam Mobin; Writing - original draft preparation: Anam Mobin; Writing — review and editing: Ekram Khan, Mirza Salim Beg, and Anam Mobin; Resources: Ekram Khan, and Mirza Salim Beg; Supervision: Ekram Khan, and Mirza Salim Beg.

\section{Compliance with ethical standards}

Conflict of interest All authors declare that they have no conflict of interest.

Open Access This article is licensed under a Creative Commons Attribution 4.0 International License, which permits use, sharing, adaptation, distribution and reproduction in any medium or format, as long as you give appropriate credit to the original author(s) and the source, provide a link to the Creative Commons licence, and indicate if changes were made. The images or other third party material in this article are included in the article's Creative Commons licence, unless indicated otherwise in a credit line to the material. If material is not included in the article's Creative Commons licence and your intended use is not permitted by statutory regulation or exceeds the permitted use, you will need to obtain permission directly from the copyright holder. To view a copy of this licence, visit http://creativecommons .org/licenses/by/4.0/.

\section{References}

1. Said A, Pearlman WA (1996) A new, fast, and efficient image codec based on set partitioning in hierarchical trees. IEEE Trans Circuits Syst Video Technol 6(3):243-250

2. Nguyen TV, Cosman PC, Milstein LB (2014) Double-layer video transmission over decode-and-forward wireless relay networks using hierarchical modulation. IEEE Trans Image Process 23(4):1791-1804

3. Barmada B, Ghandi MM, Jones EV, Ghanbari M (2005) Prioritized transmission of data partitioned H. 264 video with hierarchical QAM. IEEE Signal Process Lett 12(8):577-580

4. Ghandi MM, Ghanbari M (2006) Layered H. 264 video transmission with hierarchical QAM. J Vis Commun Image Represent 17(2):451-466

5. Chang SH, Rim M, Cosman PC, Milstein LB (2012) Optimized unequal error protection using multiplexed hierarchical modulation. IEEE Trans Inf Theory 58(9):5816-5840

6. Khan MA, Moinuddin AA, Khan E, Ghanbari M (2016) Optimized unequal error protection of embedded video bitstream using adaptive-hierarchical QAM. Multimed Tools Appl 75(23):15729-15762

7. Mobin A, Moinuddin AA, Khan E, Beg MS (2016) Optimized multi-layered unequal error protection of SPIHT coded images using 64-HQAM. In: 2016 IEEE international conference on advances in computing, communications and informatics (ICACCI), pp 2213-2218

8. You D, Kim DH (2016) Hierarchical modulation for client-driven selective streaming of multi-view video over AWGN channels. Sig Process Image Commun 47:152-159

9. Alatan AA, Zhao M, Akansu AN (2000) Unequal error protection of SPIHT encoded image bit streams. IEEE J Sel Areas Commun 18(6):814-818

10. Sprljan N, Mrak M, Izquierdo E (2005) A fast error protection scheme for transmission of embedded coded images over 
unreliable channels and fixed packet size. In: Proceedings (ICASSP'05) IEEE international conference on acoustics, speech, and signal processing, vol 3, pp. iii-741

11. Thomos N, Boulgouris NV, Strintzis MG (2005) Wireless image transmission using turbo codes and optimal unequal error protection. IEEE Trans Image Process 14(11):1890-1901

12. Cao $L$ (2007) On the unequal error protection for progressive image transmission. IEEE Trans Image Process 16(9):2384-2388

13. Mhamdi M, Zribi A, Perrine C, Pousset $Y$ (2019) Efficient multiple concatenated codes with turbo-like decoding for UEP wireless transmission of scalable JPEG 2000 images. IEEE Access 7:6327-6336

14. Rahnavard N, Pishro-Nik H, Fekri F (2007) Unequal error protection using partially regular LDPC codes. IEEE Trans Commun 55(3):387-391

15. Ahmad S, Hamzaoui R, Al-Akaidi MM (2010) Unequal error protection using fountain codes with applications to video communication. IEEE Trans Multimed 13(1):92-101

16. Yuan L, Li H, Wan Y (2016) A novel UEP fountain coding scheme for scalable multimedia transmission. IEEE Trans Multimed 18(7):1389-1400

17. Yu X, Li Y, Yang W, Sun Y (2016) Design and analysis of unequal error protection rateless spinal codes. IEEE Trans Commun 64(11):4461-4473

18. Liang H, Liu A, Cheng F, Liang X (2019) Rateless polar-spinal coding scheme with enhanced information unequal error protection. IEEE Access 7:145996-146004

19. Ghandi MM, Barmada B, Jones EV, Ghanbari M (2006) H. 264 layered coded video over wireless networks: channel coding and modulation constraints. EURASIP J Adv Signal Process 2006(1):085870

20. Pei Y, Modestino JW (2007) Cross-layer design for video transmission over wireless Rician slow-fading channels using an adaptive multiresolution modulation and coding scheme. EURASIP J Adv Signal Process 1:086915

21. Arslan SS, Cosman PC, Milstein LB (2009) Progressive source transmissions using joint source-channel coding and hierarchical modulation in packetized networks. In: GLOBECOM 2009-2009 IEEE global telecommunications conference, pp 1-6

22. Arslan SS, Cosman PC, Milstein LB (2011) Coded hierarchical modulation for wireless progressive image transmission. IEEE Trans Veh Technol 60(9):4299-4313

23. Khan MA, Moinuddin AA, Khan E, Ghanbari M (2016) Optimized cross-layered unequal error protection for SPIHT coded wireless video transmission. IEEE Trans Broadcast 62(4):876-889

24. You D, Kim DH (2020) Combined inter-layer FEC and hierarchical QAM for stereoscopic 3D video transmission. Wirel Pers Commun 110(3):1619-1636

25. Deng K, Yuan L, Wan Y, Pan J (2018) Optimized cross-layer transmission for scalable video over DVB-H networks. Sig Process Image Commun 63:81-91

26. Hosany MA (2020) Error performance analysis of an unequal error protection system for the JPEG 2000 and H. 265 standards over wireless channels. Wirel Pers Commun

27. Berrou C, Pyndiah R, Adde P, Douillard C, Le Bidan R (2005) An overview of turbo codes and their applications. In: The IEEE European conference on wireless technology, pp 1-9

28. Long X, Chen Q, Xiao P, Wu J (2012) On the design of PS-RCPT codes for LTE system. In: 2012 IEEE global communications conference (GLOBECOM), pp 4238-4243

29. Proakis J, Salehi M (2008) Digital communications, 5th edn. McGraw-Hill, London

30. Hore A, Ziou D (2010) Image quality metrics: PSNR vs. SSIM. In: 2010 20th IEEE international conference on pattern recognition, pp 2366-2369

Publisher's Note Springer Nature remains neutral with regard to jurisdictional claims in published maps and institutional affiliations. 